\title{
EVOLVE : A Visualization Tool for Multi-Objective Optimization Featuring Linked View of Explanatory Variables and Objective Functions
}

\author{
Maki Kubota, Takayuki Itoh \\ Ochanomizu University \\ Tokyo, Japan \\ \{makinosuke, itot\}@itolab.is.ocha.ac.jp
}

\author{
Shigeru Obayashi, Yuriko Takeshima \\ Tohoku University \\ Miyagi, Japan \\ obayashi@ifs.tohoku.ac.jp, \\ takeshima@vis.ifs.tohoku.ac.jp
}

\begin{abstract}
Multi-objective optimization tools have been applied in various academic and industry fields. It is often difficult to optimize all the objectives since they often cause trade-offs. It is also difficult to figure what kinds of trade-offs actually cause. We think visualization of multi-objective optimization results assists users to intuitively understand the distributions of their solutions. This paper proposes a visualization of explanatory variable and objective function spaces in the separate views, so that users can easily understand their relevancy. Also, our tool features the linkage mechanism between the two views. When a user selects certain ranges of the values by a mouse click operation, the tool highlights all the corresponding individuals. This mechanism is useful for users to narrow down the results. We expect the tool assists the understanding of the behavior of the optimization processes and improvement of the future processes. (Abstract)
\end{abstract}

Keywords--multi-objective optimization; explanatory variable; objective function; parallel coordinate plots; scatterplots (key words)

\section{INTRODUCTION}

Optimization problem is finding one or more solutions under which was placed under restrictions. In the real world, there are few single-objective optimization problems: Our daily problems usually contain more than one objective functions which have complicated or tradeoff relationships. Multiple-objective optimization is a problem that attempts to optimize multiple objective functions simultaneously. In other words, we handle multi-objective optimization problems formalized as follows:

$$
\text { Minimize }\left\{f_{1}(\xi), f_{2}(\xi), \ldots, f_{\mathrm{m}}(\xi)\right\}
$$

$$
\text { Subject to } \xi \in \Sigma
$$

The problem involves $\mathrm{m}(2)$ conflicting objective functions $f_{\mathrm{i}}: \mathrm{R}_{\mathrm{n}} \rightarrow \mathrm{R}$ that we want to minimize simultaneously. The decision (variable) vectors $\xi=\left(\xi_{1}, \xi_{2}, \ldots, \xi_{\mathrm{n}}\right) \mathrm{T}$ belong to the nonempty feasible region $\Sigma \subset \mathrm{R}_{\mathrm{n}}$. In this general problem formulation we do not fix the types of constraints forming the feasible region. Objective vectors are images of decision vectors and consist of objective (function) values $\mathrm{z}=f(\xi)=$ $\left(f_{1}(\xi), f_{2}(\xi), \ldots, f_{\mathrm{m}}(\xi)\right) \mathrm{T}$. Furthermore, the image of the feasible region in the objective space is called a feasible objective region $\mathrm{Z}=f(\Sigma)$.

Problems of multi-objective optimization are often complicated since these objective functions often bring trade-offs. Here, explanatory variables and objective functions are multi-dimensional values. Therefore, multidimensional data visualization tools are useful for observation of explanatory variables and objective functions.

This paper presents EVOLVE (Explanatory Variable and Objective function Linked Visualization Environment), a visualization tool which displays both explanatory variables and objective functions for multi-objective optimization. The tool splits the visualization window into left and right regions, and visualizes explanatory variables in the left region, and objective functions in the right regions. It also features a user interface to narrow down and highlight the ranges of explanatory variables and/or objective functions. It applies a linkage mechanism that highlights the particular parts in the objective function space, when a user specifies corresponding parts in the explanatory variable space, and also highlights the particular parts in the explanatory variable space, when a user specifies corresponding parts in the objective function space. Such mechanism has been already well known as "Coordinate View" or "Linked View" in the visualization research field; however, it has not been well applied to the visualization for multi-objective optimization systems. This paper demonstrates this mechanism is useful for observation of multi-objective optimization problems. 


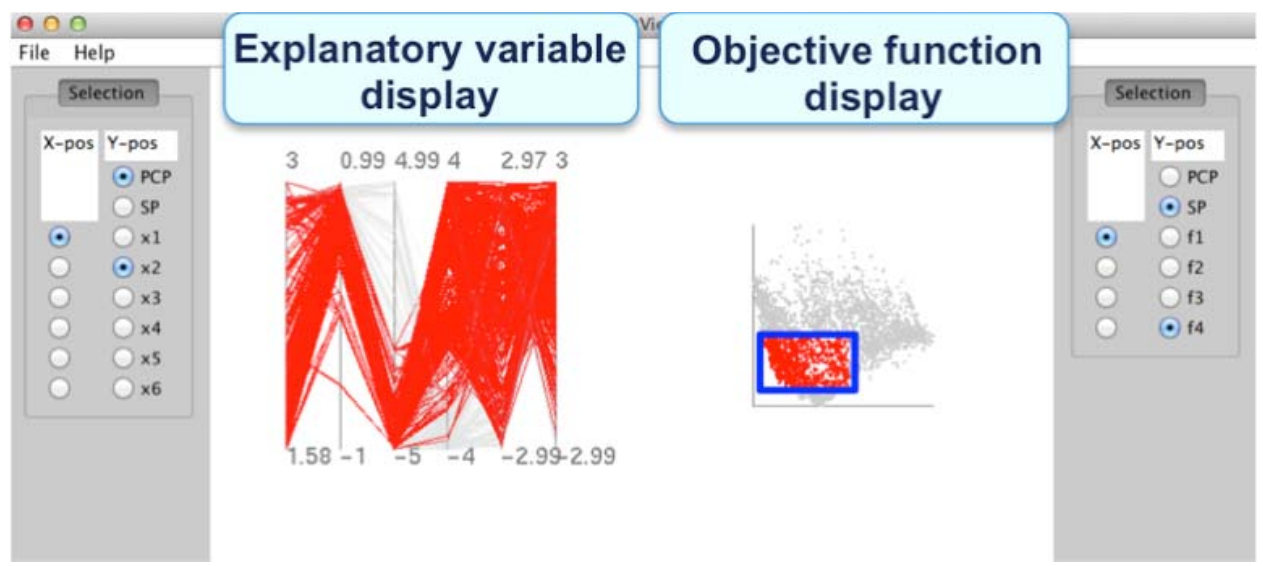

Figure 1. Overview of the EVOLVE.

\section{RELATED WORK}

\section{A. Multi-dimensional Data Visualization}

Multi-dimensional data visualization tools are useful for observation of explanatory variables and objective functions. This section introduces several famous multi-dimensional data visualization tools. Scatterplot is the most frequently used multi-dimensional data visualization tool. "Scatterplot matrix" has been also well used to visualize three or more dimensions in a single display space. Effective scatterplotbased interaction tools [1] have been recently also presented for exploration of multi-dimensional spaces. Several other visualization techniques attempt to directly represent three or more dimensions. Glyph-based techniques [2] are Parallel Coordinate Plot (PCP) [3] are typical techniques. These techniques have their own pros and cons. Scatterplot has been already widely used because of its simplexes, and is useful for relatively large number of samples. On the other hand, PCP has an advantage of simultaneous visualization of three or more dimensions. Such techniques should be selectively and alternatively applied.

\section{B. Visualization for Multi-objective Optimization}

Distributions of explanatory variables and objective functions are often very complicated, and therefore several related studies applied visualization techniques for the observation. Eddy et al. [4] presented "Cloud Visualization" which applies a scatterplot for visualization of Pareto solutions. Obayashi et al. [5] presented a technique applying the Self Organization Map (SOM) to cluster and visualize Pareto solutions. Agrawal et al. [6] presented a technique to visualize Pareto frontier regions. Pryke et al. [7] presented a similar technique using a heatmap. These tools do not directly represent explanatory variables.

\section{VISUALIZATION OF EXPLANATORY VARIABLES AND OBJECTIVE FUNCTIONS}

This section introduces features implemented to EVOLVE for visualization of explanatory variables and objective functions.

\section{A. Selection of Visualization Tool}

Fig. 1 shows a snapshot of EVOLVE for the visualization of explanatory variables and objective functions. This tool splits the window into the left and right regions, and applies multidimensional data visualization tools to the both regions. Our implementation assigns explanatory variables to the left region, and objective functions to the right region.

Multi-dimensional data visualization tools have their advantages and disadvantages. Therefore we have concluded to implement multiple visualization tools and selectively apply them to explanatory variables and objective functions. Our current implementation manually switches Parallel Coordinate Plot (PCP)[1] and twodimensional scatterplot. The left and right ends of the window feature buttons to select the visualization tools, as shown in Fig. 1.

\section{B. Variable Selection}

Explanatory variables may be very high dimensional in many optimization problems. While scatterplots just represents two or three-dimensional values, Parallel Coordinate Plot can represent relatively higher dimensional values. However, Parallel Coordinate Plot needs large display spaces for such high dimensional values. Moreover, Parallel Coordinate Plot often causes cluttering of polylines, which prevents the understanding of the visualized datasets. Our implementation solves such problems by only displaying automatically selected important explanatory variables. We 

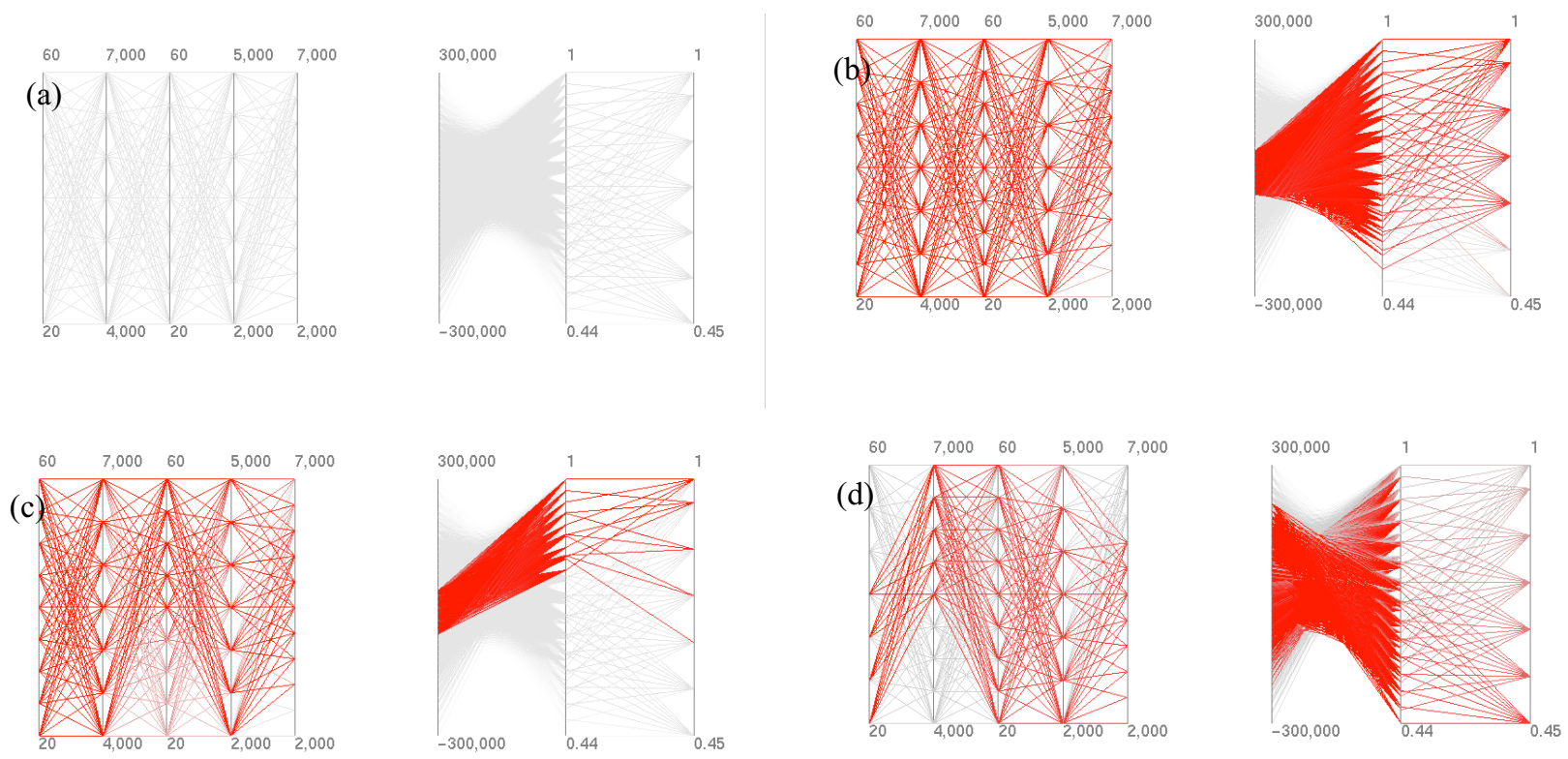

Figure 2. (a) specified non-selected display, (b) specified $f_{1}$ is close to 0 , (c) specified $f_{1}$ is close to 0 and $f_{2}$ values are sufficiently large and (d) specified low $\mathrm{n}_{1}$ value and high $\mathrm{n}_{2}$ value cause $f_{3}$ is lower.

apply the redundant feature elimination method [2] presented by Kawakubo et al.

\section{Interaction for Region Interest Specification}

EVOLVE features a user interface to select particular samples by drawing rectangular regions like a blue rectangle in Fig. 1. The linkage mechanism implemented on EVOLVE highlights particular parts in the objective function space when a user specifies corresponding parts in the explanatory variable space. Similarly, it highlights the particular parts in the explanatory variable space when a user specifies corresponding parts in the objective function space. This linkage mechanism enables the user to narrow down in both the explanatory variable and objective function spaces. The set of red polylines in Fig. 1 is an example of the highlighting result after the selection of blue rectangular region of interest. This kind of linked view mechanisms have not been well applied to visualization of multi-objective optimization in the previous studies $[3,4]$.

\section{Polyline Clustering}

EVOLVE features a clustering algorithm to divide the polylines into user-specified number of clusters. Our current implementation simply treats $\mathrm{n}$ explanatory variables and corresponding $\mathrm{m}$ objective function values as a $(\mathrm{m}+\mathrm{n})$ dimensional vector, and applies the k-means algorithm. EVOLVE displays the clustering result as a set of colored polylines.

\section{CASE StUdy}

This section introduces two case studies applying EVOLVE. The former one is a visualization of modeled problem on party fee and profit. The latter one is a visualization of fluid dynamics simulation results.

\section{A. Example 1: Party Fee Problem}

This section introduces a party fee problem as a simple virtual problem. Here, we describe the five explanatory variables as follows:

- $\quad$ the numbers of male and female participants as $n_{1}$ and $n_{2}$,

- $\quad$ the party fees of male and female participants as $p_{1}$ and $p_{2}$, and

- $\quad$ the fee per participant paid for the party venue as $c$.

This section also describes the following objective functions $f_{1}$ to $f_{3}$ :

- $f_{1}$ : Profit of the party organizer. We modeled it as the following equation:

$$
f_{1}=\left(p_{1} n_{1}+p_{2} n_{2}\right)-c\left(n_{1}+n_{2}\right)
$$

- $f_{2}$ : Satisfaction of male participants. We modeled it as the following equation with two functions $g_{1}$ and $g_{2}$ :

$$
f_{2}=1.0-\left(g_{1}+g_{2}\right)
$$


Here, $g_{1}(\mathrm{c})$ is a monotone decreasing function representing the complaint to the cuisine, and $g_{2}\left(n_{1}-n_{2}\right)$ is a monotone increasing function representing the complaint to the lack of female participants.

- $f_{3}$ : Satisfaction of female participants. We modeled it as the following function with a function $g_{3}$ :

$$
f_{3}=1.0-g_{3}
$$

Here, $g_{3}(c)$ is a monotone decreasing function representing the complaint to the cuisine.

The set of above three functions contain a trade-off, which $f_{2}$ and $f_{3}$ get worse while $f_{1}$ gets better due to the increase of $c$. At the same time, increase of $n_{1}$ brings the improvement of $f_{1}$, while the increase of $n_{1}-n_{2}$ brings the reduction of $f_{2}$. This section introduces the visualization of explanatory variables and objective functions by PCP, where explanatory variables are drawn in the order of $n_{1}, p_{1}, n_{2}, p_{2}, c$, and objective functions are drawn in the order of $f_{1}, f_{2}, f_{3}$.

Fig. 2(a) shows the distribution of explanatory variables and objective functions. Fig. 2(b) shows an example of highlighting particular polylines in red, with an operation which specifies polylines whose $f_{1}$ values are close to zero. This result denotes that the highlighted polylines concentrates at the center of the axis of $f_{1}$, while they disperse on the other axes. Fig. 2(c) shows the next result with the operation which specifies polylines whose $f_{2}$ values are sufficiently large. This figure denotes that the highlighted polylines on the axis of $n_{2}$ concentrate to the upper side of the axis. It denotes that sufficient number of female participants are desirable to improve the satisfactory of male participants.

In the above examples, we could narrow the ranges of explanatory variables while narrowing the ranges of objective functions. Fig. 2(d) shows an example which specifies polylines whose $n_{1}$ values are sufficiently small.

\section{B. Airplane Shape Design}

This section introduces a case study applying EVOLVE to the optimization of airplane shape design. Shape design is a process to find a point in the design variable space that matches with the given point in the objective function space. However, this is very difficult to find. Our experiment designed a whole wing shape applying 72 design variables. Fig. 2 shows the most important 6 variables to define the whole wing shape. Improvement of transport efficiency is a very important issue in the airplane development field. Increase of the routes, flights, or size of the bodies can be considered for expansion of the transportation volume. On the other hand, a lot of attention is needed while developing new bodies of airplanes to archive small resistance. Large scale SST (Supersonic transport) has a problem that noise phenomenon, called sonic boom, accompany that air pressure rise reach the ground by supersonic flight. Therefore, supersonic flight is limited in the marine flight. It is necessary to evaluate the cruising speed of the two kinds of transonic and supersonic.

We designed various shapes of the wings by varying the design variables, and evaluated them applying the following four objective functions:

- Drag coefficient during transonic cruise.

- Drag coefficient during supersonic cruise.

- Bending moment at the wing root during supersonic cruise.

- $\quad$ Pitching moment during supersonic cruise.

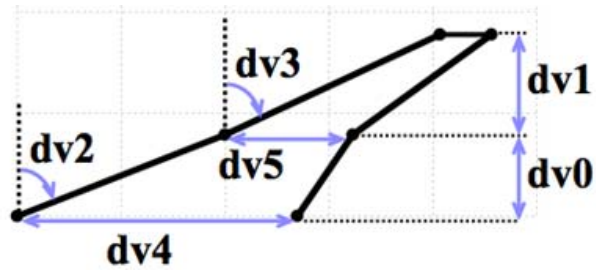

Figure 3. Representative variables for wing shape design.

These objective functions are to be minimized in this optimization problem. We applied a multi-objective genetic algorithm to solve the problem, where the population size was 64 , and the evolution was computed for 75 generations until all individuals become non-dominated. We used the redundant features elimination method described in Section 3.2, and selected the 6 variables shown in Fig. 3. These variables, which determine platform design, are dominant for the wing performance. Here, dv0 and dv1 determine the span lengths of the inboard and outboard wing panels, respectively. dv2 and dv3 correspond to leading-edge sweep angles. dv4 and dv5 are root-side chord lengths. Other design variables determine wing camber and wing twist. Fig. 3 shows only important variables for wing shape design.

\section{1) Findings from the selecting system}

This subsection shows the results of the visualization. Fig. 4 (Left) shows examples of the visualization result where a user specified the ranges of objective functions: smaller drag coefficient during supersonic cruise, and larger bending moment at the wing root. The result shows that the ranges of explanatory valuables are quite similar while specifying the two kinds of ranges of objective functions and also these two objective functions have trade-off relationship. Fig. 4(Right) shows another result where a user specified the ranges of objective functions: smaller drag coefficient during supersonic cruise and pitching moment during supersonic cruise. That shows this aspect ratio is high because $\mathrm{dv} 0$ is lager and $\mathrm{dv} 5$ and $\mathrm{dv} 6$ are smaller. Additionally, dv2 and dv3 are smaller. We have already found that drag coefficient during transonic and supersonic cruise is low while aspect ratio is high, in our previous study with this dataset. In addition, we found that pitching moment during supersonic cruise gets smaller while sweep 
angles are smaller, by using EVOLVE. It is new knowledge for us that these phenomena is simultaneously observed in the result shown in Fig. 4(Right).

\section{2) Findings from clustering}

Fig. 5 shows results of the clustering result where a user specified the ranges of objective functions: smaller drag coefficient during transonic cruise (Fig. 5(Upper-left)), smaller drag coefficient during supersonic cruise (Fig. 5(Upper-right)), smaller bending moment at the wing root (Fig. 5(Lower-left)), and smaller pitching moment at the wing root (Fig. 5(Lower-right)). Each of results includes two colors. As we mentioned before there are trade off relationship between two drag coefficients and two moments. However, in this section, we could find new relationships, which are drag coefficient during transonic cruise and pitching moment at the wing root, drag coefficient during supersonic cruise and smaller bending moment at the wing root.

\section{CONCLUDING REMARKS AND FUTURE WORK}

This paper presented EVOLVE, a visualization tool which features a linkage mechanism between explanatory variables and objective functions. The tool improves visibility of coherence between them, and assists the understanding the numeric distributions of multi-objective optimization problems.

Following are our potential future issues.

\section{1) Extension and improvements of visualization tools}

There have been various improved multi-dimensional data visualization techniques, and many of them can be applied to EVOLVE. We would like to apply more interactive techniques for scatterplot [1] so that we can easily select interesting dimensions to be visualized. Also, dimension reordering techniques for Parallel Coordinate Plot should be useful to improve the visibility of numeric coherence among the dimensions. Moreover, we would like to implement other multi-dimensional data visualization techniques in addition to scatterplot and Parallel Coordinate Plot.

\section{2) Linkage with optimization schemes}

Our current implementation supposes that the optimization system is disconnected from EVOLVE, and just receives the final optimization results as data files. This processing flow is just based on the specification of our current project which deals with the confidential datasets of real industry. On the other hand, it should be helpful if EVOLVE is connected to optimization schemes to monitor the progress of iterative calculations. We think this mechanism can assist the users to understand how the optimization schemes approaches to Pareto solutions. We would like to implement optimization schemes which can be connected to EVOLVE.

\section{3) More case studies and evaluations}

EVOLVE does not depend on any applications, and therefore can be applied to various scientific and industrial fields. We would like to apply EVOLVE to more various fields, and review the effectiveness of the visualization results by subjective user evaluations.

\section{REFERENCES}

[1] N. Elmqvist, P. Dragicevic, and J. Fekete, "Rolling the Dice: Multidimensional Visual Exploration using Scatterplot Matrix Navigation," IEEE Transactions on Visualization and Computer Graphics, 14(6), 1141-1148, 2008.

[2] D. A. Keim, "Information Visualization and Visual Data Mining," IEEE Transactions on Visualization and Computer Graphics, 8(1), pp. 1-8, 2002.

[3] A. Inselberg, and B. Dimsdale, "Parallel Coordinate. A Tool for Visualizing Multi-Dimensional Geometry," IEEE Visualization, 361-370, 1990.

[4] J. Eddy, and K. Lewis, "Visualization of Multidimensional Design and Optimization Using Cloud Visualization," ASME Design Engineering Technical Conferences, DETC02/DAC-2006.

[5] S. Obayashi, and D. Sasaki, "Visualization and data mining of Pareto solutions using self-organizing map," Lecture Notes in Computer Science 2632: Evolutionary MultiCriterion Optimization, 796-809, 2003.

[6] G. Agrawal, K. Lewis, K. Chugh, C.H. Huang, S. Parashar, and C. L. Bloebaum, "Intuitive Visualization of Pareto Frontier for Multi-Objective Optimization in N-Dimensional Performance Space," Tenth AIAA/ISSMO Multidisciplinary Analysis and Optimization Conference, Albany, AIAA2004-4434, 2004.

[7] A. Pryke, S. Mostaghim, and A Nazemi, "Heatmap Visualization of Population Based Multi Objective Algorithms," Evolutionary multi-criterion optimization. Evolutionary Multi-Criterion Optimization, 361-375, 2007. 

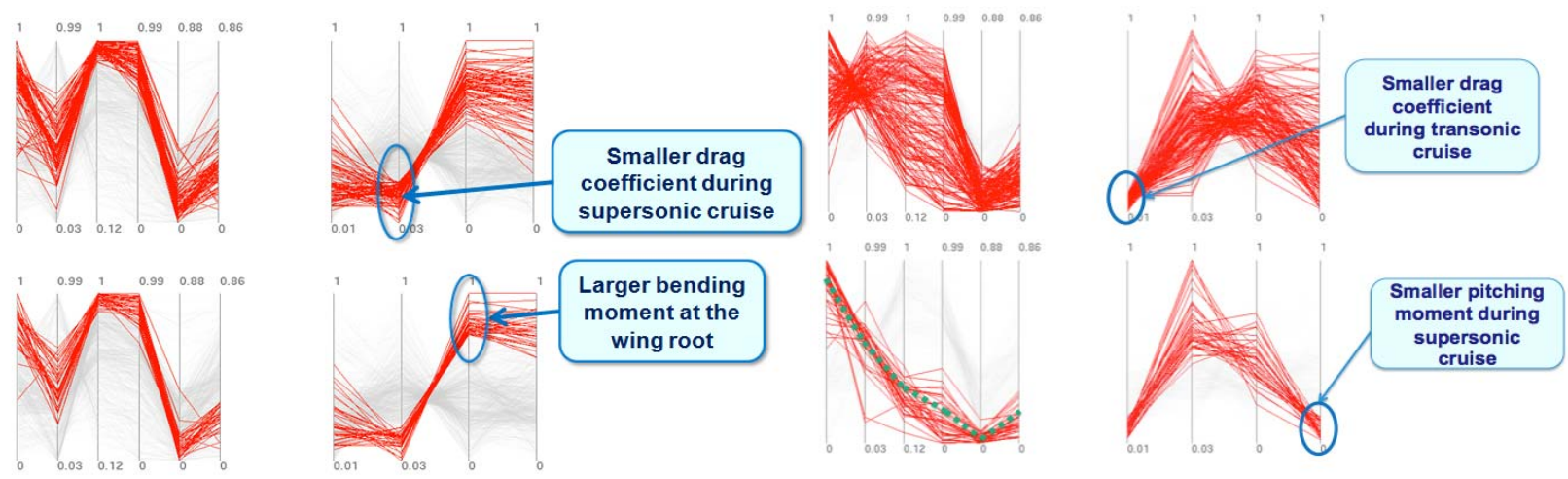

Figure 4. (Left) Specified smaller drag coefficient during supersonic cruise, and larger bending moment at the wing root (Right) Specified smaller drag coefficient during transonic cruise and smaller pitching moment during supersonic cruise.
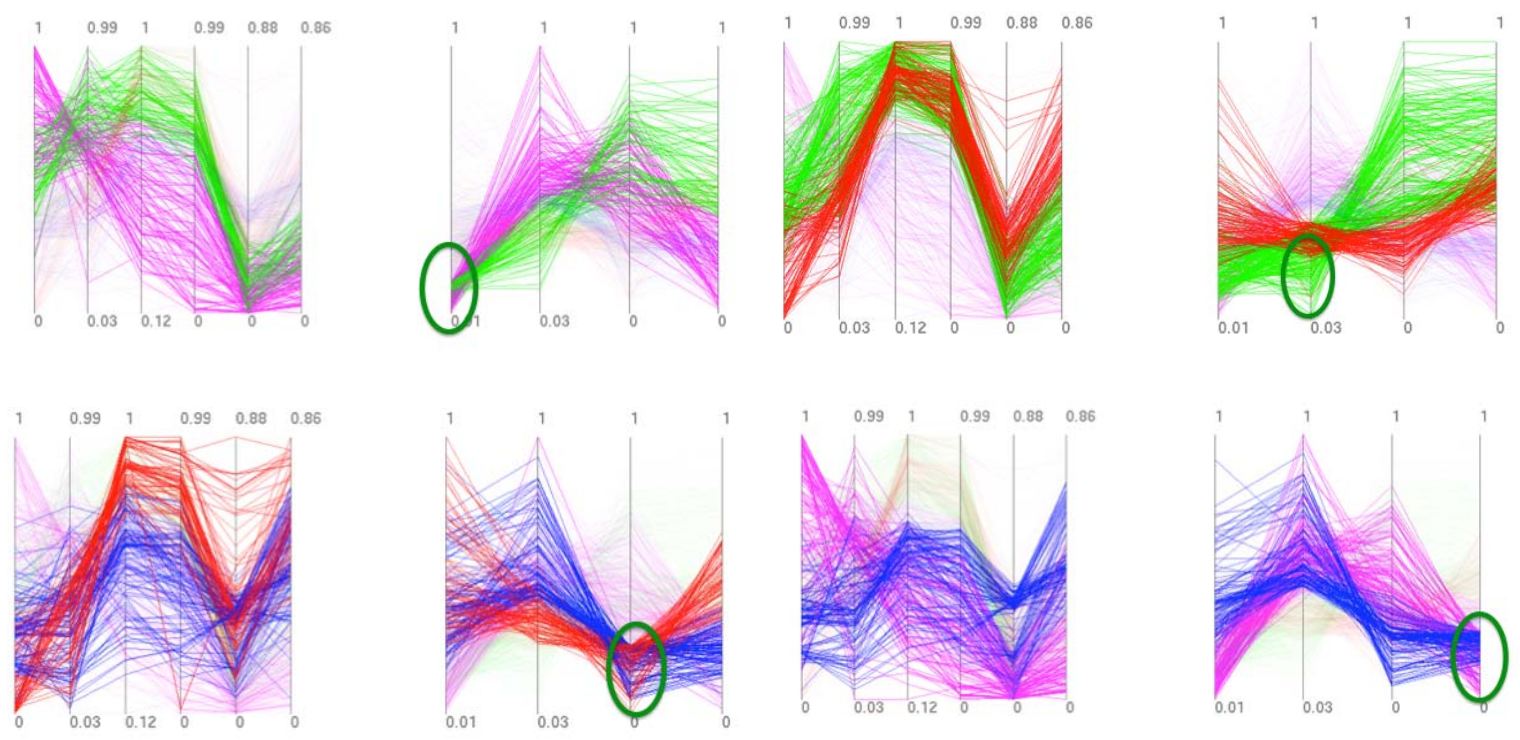

Figure 5. Clustered View. (Upper-left) Smaller drag coefficient during transonic cruise. (Upper-right) Smaller drag coefficient during supersonic cruise. (Lower-left) Smaller bending moment at the wing root. (Lower-right) Smaller pitching moment at the wing root. 